\title{
Efficacy of dydrogesterone on treating recurrent miscarriage and its influence on immune factors: a systematic review and meta-analysis
}

\author{
Hongyu Guo, Qibin Lu
}

Department of Gynaecology, Affiliated Hospital of Nanjing University of Chinese Medicine, Jiangsu Province Hospital of Chinese Medicine, Nanjing, China

Contributions: (I) Conception and design: Both authors; (II) Administrative support: Q Lu; (III) Provision of study materials or patients: Both authors; (IV) Collection and assembly of data: Both authors; (V) Data analysis and interpretation: Both authors; (VI) Manuscript writing: Both authors; (VII) Final approval of manuscript: Both authors.

Correspondence to: Qibin Lu. Department of Gynaecology, Affiliated Hospital of Nanjing University of Chinese Medicine, Jiangsu Province Hospital of Chinese Medicine, Nanjing 210049, China. Email: fsyy00601@njucm.edu.cn.

\begin{abstract}
Background: This study aimed to explore the clinical efficacy of dydrogesterone in treating recurrent spontaneous abortion (RSA), analyze the influence of dydrogesterone on cellular immune factors, and provide evidence for clinical medication.

Methods: We used the China National Knowledge Infrastructure (CNKI) platform, Wanfang Data resource, PubMed, Web of Science, and Embase database to conduct a literature search to screen clinical studies published between 2005 and 2021 concerning dydrogesterone treatment for RSA. Stata 16.0 was used for meta-analysis and sensitivity analysis, and Begg's funnel chart was used to test publication bias.

Results: Only 13 studies, which included a total of 2,454 RSA patients, met the study inclusion criteria. The experimental group was treated with dydrogesterone, and the control group was treated with progesterone, human chorionic gonadotropin (hCG), placebo, or active immunization. Meta-analysis showed that the pregnancy success rate of the experimental group was higher than the control group, and the adverse reaction rate was lower than the control group. In addition, subgroup analysis also revealed that the experimental group had a higher pregnancy success rate than the control group and a lower adverse reaction rate. Levels of progesterone and hCG in the experimental group were dramatically higher than the control group after treatment. The experimental group also had higher levels of interleukin 4 (IL-4) and interleukin 10 (IL-10) than the control group, while levels of interferon-gamma (IFN- $\gamma$ ) were lower.
\end{abstract}

Discussion: Dydrogesterone, a safe and effective synthetic progesterone drug, had a significant clinical effect on RSA and effectively improved hormone levels and related cellular immune factors in RSA patients.

Keywords: Recurrent miscarriage; dydrogesterone; hormones; immune factors; meta-analysis

Submitted Aug 25, 2021. Accepted for publication Oct 09, 2021.

doi: 10.21037/apm-21-2605

View this article at: https://dx.doi.org/10.21037/apm-21-2605

\section{Introduction}

Recurrent spontaneous abortion (RSA) refers to the occurrence of 3 or more pregnancy losses within 28 weeks of falling pregnant with the same person. Recommendations have been made in China to make changes to the medical definition to include 2 consecutive miscarriages (1-3).
RSA, also called habitual abortion, has an incidence rate of about $1-5 \%$ (4) and is positively correlated with the number of miscarriages. After more than three consecutive spontaneous abortions, the miscarriage rate is about $40 \%$, and this increases with age $(5,6)$. At present, most studies have shown that the primary cause of RSA is closely 
related to immune factors, followed by a range of other causes, including antiphospholipid syndrome, infection, thrombosis, hormones, anatomy, genetics, psychology, and environment $(7,8)$.

In cases where RSA is due to progesterone insufficiency, progesterone or dydrogesterone is usually used in clinical treatment (9). Progesterone has been used clinically as a natural progestin since the 1960s, but there are side effects, including drowsiness, headache, nausea, and vomiting (10). Recent studies have shown that progesterone usage, such as vaginal progesterone treatment, can ameliorate RSA in some pregnant women, although potential safety concerns remain (11). Dydrogesterone, the synthetic form of progesterone, has a similar molecular structure to progesterone but has fewer side effects and superior bioavailability, with 5.6 times the bioavailability of progesterone (12). Dydrogesterone and its main active metabolites are highly selective for progesterone receptors (13) and cannot bind to androgen receptors, which greatly reduces the risk of virilization of female fetuses (14). Due to the high bioavailability and receptor selectivity of dydrogesterone, the dose required clinically is also markedly less. It has been estimated that the oral dose of dydrogesterone is 10-20 times lower than progesterone (15). Dydrogesterone has been found to be an effective treatment method for RSA, with an even better clinical efficacy than progesterone (16). Because cellular immune effect is closely related to the development of RSA, proinflammatory and anti-inflammatory cytokines have a crucial influence on the success or failure of pregnancy. Dydrogesterone can participate in the immune process by regulating T-helper lymphocytes to sustain the pregnancy, exerting an important function in preventing and treating RSA $(13,17,18)$. Based on the above, this study aimed to explore the clinical efficacy of dydrogesterone on RSA and its influence on immune factors by conducting a meta-analysis of randomized controlled trials (RCTs) of dydrogesterone in the treatment of RSA. We systematically evaluated the clinical efficacy of dydrogesterone and changes in related immune factors, providing clinical evidence and data to validate the effectiveness of dydrogesterone in treating RSA and guiding clinical medication. We present the following article in accordance with the PRISMA reporting checklist (available at https://dx.doi.org/10.21037/apm-21-2605).

\section{Methods}

\section{Literature search strategy}

We conducted a literature search using the China National
Knowledge Infrastructure (CNKI) platform, Wanfang Data resource, PubMed, Web of Science, and Embase database. The publication period was 2005 to 2021, and the subject terms for combined search included "progesterone", "recurrent miscarriage", and "immunization".

\section{Inclusion criteria}

The following inclusion criteria were used: (I) clinical RCT studies published in medical journals in China or abroad; (II) RSA patients with 2 or more spontaneous abortions; (III) the experimental group was treated with dydrogesterone, and the control group was treated with progesterone, human chorionic gonadotropin (hCG), placebo, or active immunization; (IV) outcome indicators, including pregnancy success rate, adverse reaction rate, and expression levels of progesterone, hCG, interleukin (IL)-4, interleukin (IL)-10, interferon-gamma (IFN)- $\gamma$, and other posttreatment indicators.

\section{Exclusion criteria}

Literature was excluded for the following reasons: (I) inconsistent research design or intervention measures; (II) miscarriage caused by primary factors such as chromosomal abnormalities and genetics; (III) unclear diagnostic criteria or outcome indicators; (IV) literature without key data required for this meta-analysis that could not be obtained even after contact with the author; (V) literature of poor quality or missing important data; (VI) repeated reports or literature that could not be obtained in its original text; and (VII) case reports, systematic reviews, and animal experiments.

\section{Literature screening and data extraction}

The literature was imported and saved into EndNote software and then further rechecked manually and with software. Subsequently, two independent researchers read the original texts to screen the literature, extracting the relevant clinical data and outcome indicators. At this stage, when disputes arose, another researcher arbitrated in order to reach a consensus. Finally, the researchers summarized the original documents, including relevant information such as title, author, publication date, research design type, research object, intervention measures, and outcome indicators. 


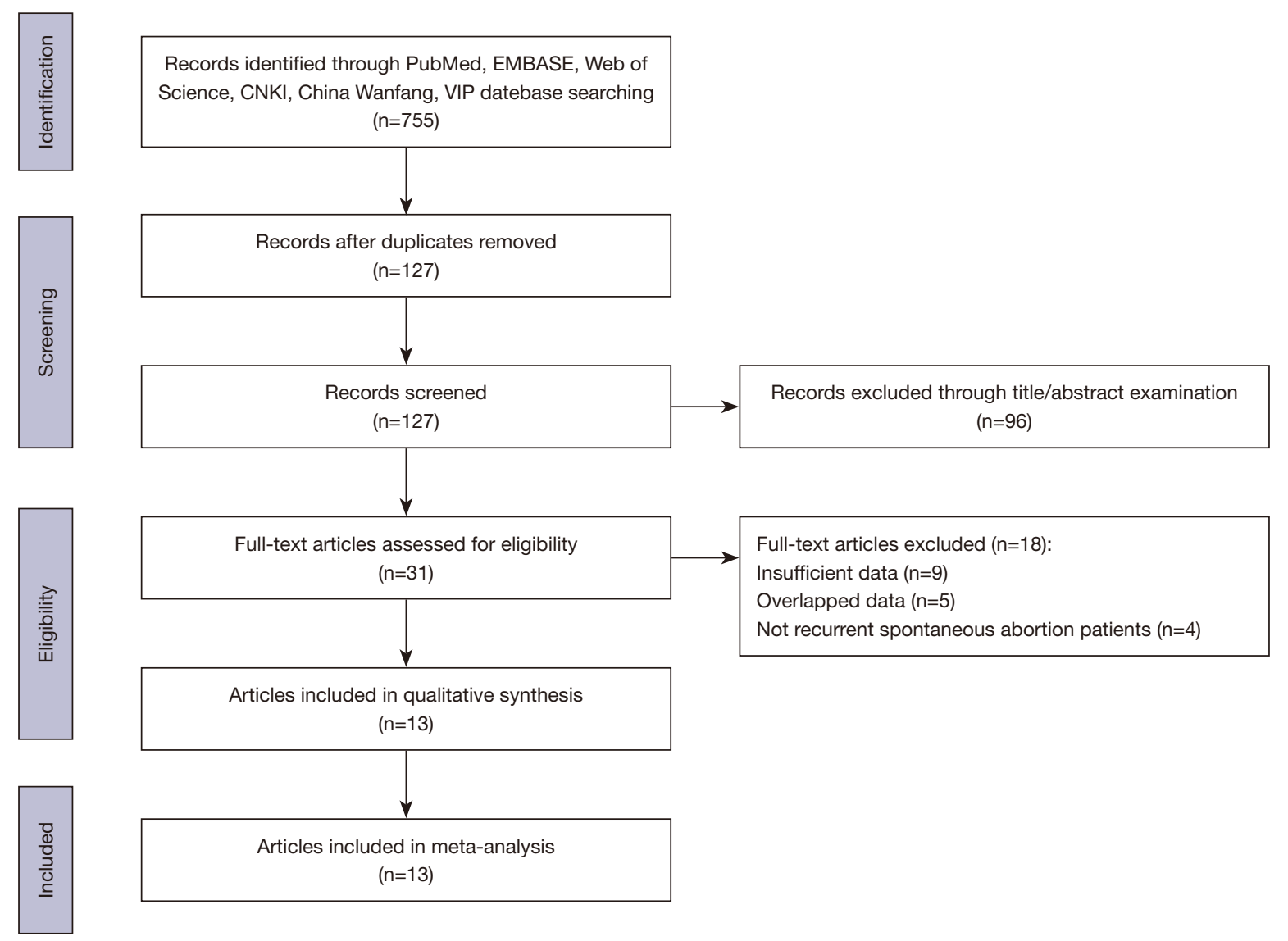

Figure 1 Document screening flow chart.

\section{Statistical analysis}

Stata 16.0 statistical software was used for the meta-analysis. The odds ratio (OR) and $95 \%$ confidence interval (CI) were used to represent dichotomous variables, and the standard mean difference (SMD) and 95\% CI were used to represent continuous variables. Statistical heterogeneity between different studies was evaluated by calculating the statistic $\mathrm{I}^{2}$. P $>0.1, \mathrm{I}^{2}<50 \%$ indicated that there was no significant difference in heterogeneity and a fixed-effect model was selected. $\mathrm{P}<0.1, \mathrm{I}^{2} \geq 50 \%$ suggested that heterogeneity between the included studies was obvious and thus a random-effects model was selected. Subgroup analysis and sensitivity analysis were performed on the causes of heterogeneity. As the final number of studies included in the meta-analysis was not less than 10 , publication bias was analyzed by making a funnel chart. $\mathrm{P}<0.05$ was considered significantly different.

\section{Results}

\section{Literature search}

A total of 755 articles were initially obtained from the database searches. After excluding duplicate articles, 127 articles remained. A further 96 articles were excluded after browsing the titles and abstracts, and 18 additional articles were excluded after reading the original texts and screening the documents. In total, 13 studies met the inclusion criteria and were included in the meta-analysis $(14,19-30)$. The literature screening process is shown in Figure 1. A total of 2,454 RSA patients were included in the studies selected for the meta-analysis, including 1,216 in the experimental group (dydrogesterone) and 1,238 in the control group (progesterone, hCG, placebo, or active immunotherapy). Key details of the included literature are shown in Table 1. 
Table 1 Key information of the included literature

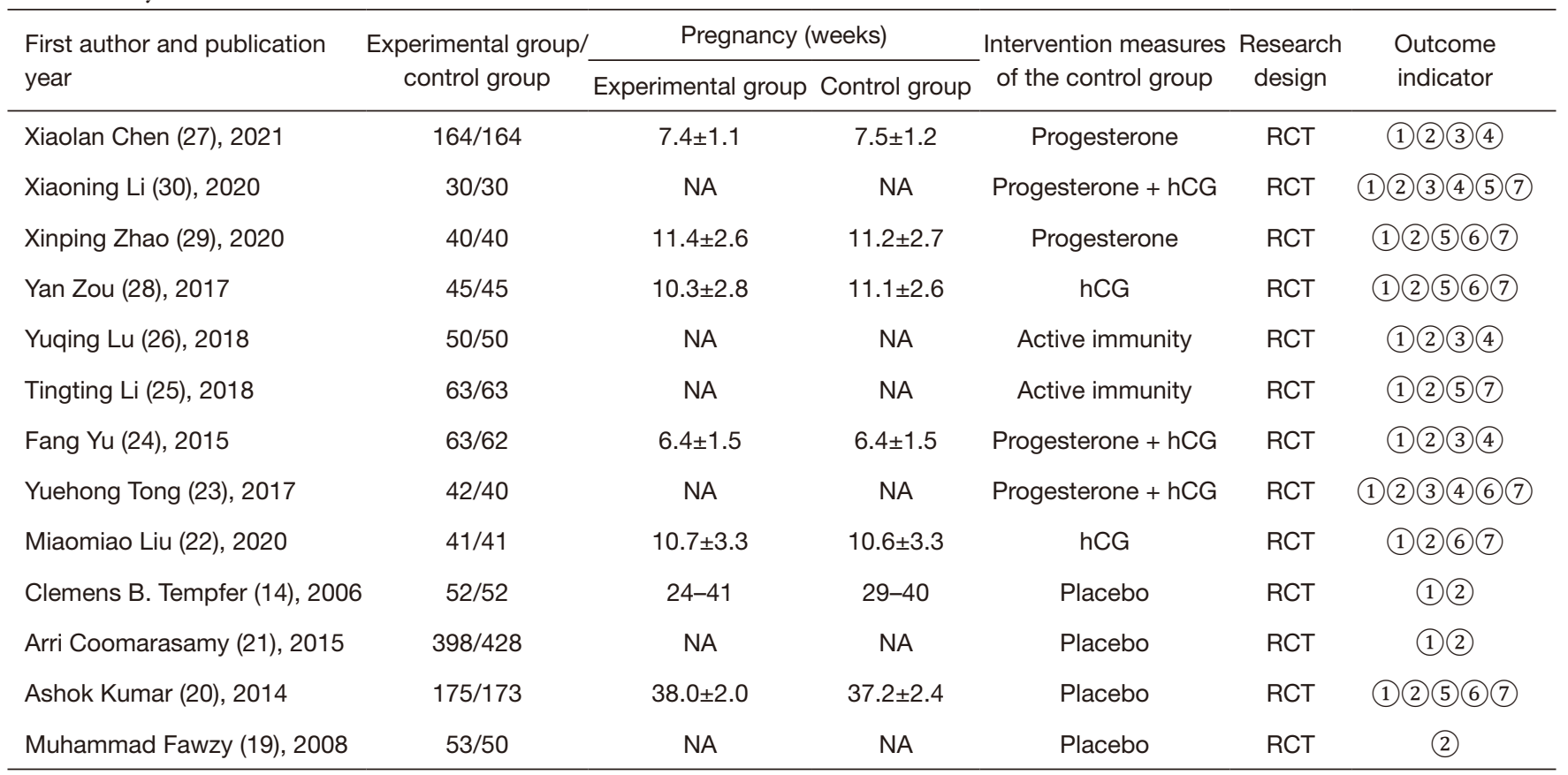

(1): pregnancy success rate; (2): adverse reaction rate; (3): progesterone level after treatment; (4): hCG level after treatment; (5): interleukin-10 level after treatment; (6): interleukin-4 level after treatment; (7): interferon level after treatment. NA, no data available; hCG, human chorionic gonadotropin; RCT, randomized controlled trial.

\section{Meta-analysis}

\section{Meta-analysis of pregnancy success rate and incidence of adverse reactions}

In order to clarify the clinical efficacy of dydrogesterone on RSA, the pregnancy success rate and adverse reaction rate of the 2 groups were analyzed. The results revealed substantial heterogeneity among the included studies $\left(\mathrm{I}^{2}=71.0 \%, \mathrm{P}=0.000\right)$, and thus a random-effects model was used for combined analysis. The pregnancy success rate of the experimental group was notably higher than the control group (OR $=4.26 ; 95 \%$ CI: 2.59-7.00, $\mathrm{P}=0.000)$ (Figure 2A). There was no obvious heterogeneity between the studies regarding adverse reactions $\left(\mathrm{I}^{2}=41.3 \%, \mathrm{P}=0.059\right)$. The results of the fixed-effect model analysis showed that the incidence of adverse reactions of the experimental group was significantly lower than the control group $(\mathrm{OR}=0.30$; 95\% CI: 0.22-0.40, $\mathrm{P}=0.000$ ) (Figure 2B).

\section{Subgroup analysis of pregnancy success rate and adverse reaction rate}

Since the intervention measures of the control group included progesterone, hCG, placebo, and active immunotherapy, a subgroup analysis of the pregnancy success rate was carried out (Figure 3A). Among them, 2 studies $(27,29)$ chose progesterone alone. As there was no significant heterogeneity between the 2 studies $\left(\mathrm{I}^{2}=0.0 \%\right.$, $\mathrm{P}=0.692$ ), we selected a fixed-effect model for analysis. The results showed that the pregnancy success rate of dydrogesterone treatment was higher than progesterone treatment alone $(\mathrm{OR}=4.47$; 95\% CI: $2.05-9.75, \mathrm{P}=0.000)$. Three studies $(23,24,30)$ used progesterone combined with hCG treatment, and a fixed-effect model $\left(\mathrm{I}^{2}=0.0 \%, \mathrm{P}=0.858\right)$ showed that the pregnancy success rate of the experimental group was higher than the control group of progesterone combined with hCG treatment $(\mathrm{OR}=5.87$; 95\% CI: $2.45-$ $14.03, \mathrm{P}=0.000)$. In 2 studies $(22,28)$, the control group was treated with hCG alone, and the results of the fixed-effect model $\left(\mathrm{I}^{2}=0.0 \%, \mathrm{P}=0.992\right)$ showed that the dydrogesterone group had a higher pregnancy success rate than the hCG group ( $\mathrm{OR}=6.25 ; 95 \% \mathrm{CI}: 2.41-16.20, \mathrm{P}=0.000)$. Two studies $(25,26)$ selected active immunotherapy, and there was homogeneity between them $\left(\mathrm{I}^{2}=0.0 \%, \mathrm{P}=0.440\right)$. The results suggested that using dydrogesterone to treat RSA had better efficiency than active immunotherapy based on the pregnancy success rate $(\mathrm{OR}=5.18$; $95 \% \mathrm{CI}: 1.99-13.43$, $\mathrm{P}=0.000)$. There were 4 studies $(18-21)$ that chose to use 
A Study

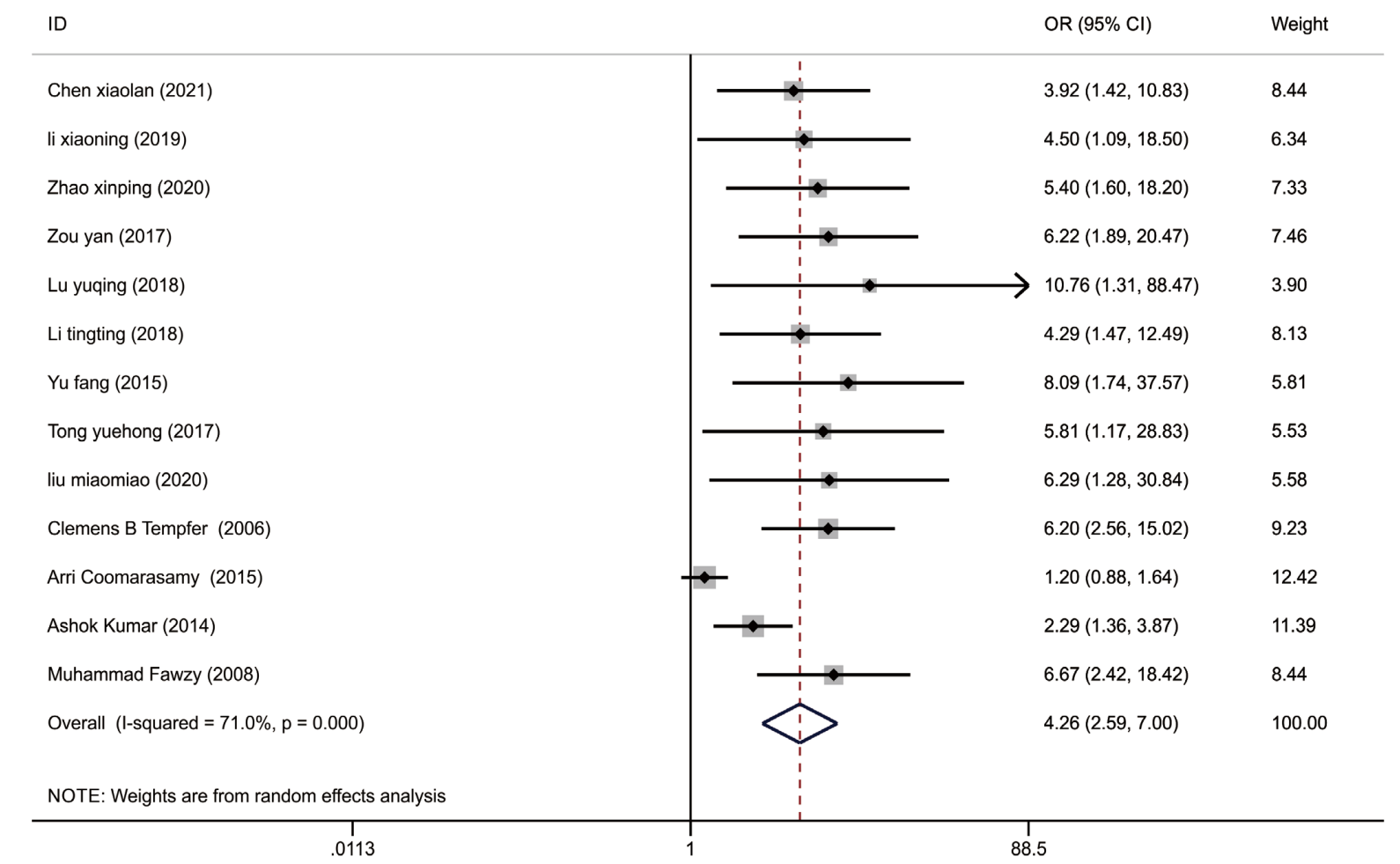

B Study

\begin{tabular}{|c|c|c|}
\hline ID & OR $(95 \% \mathrm{Cl})$ & Weight \\
\hline Chen xiaolan (2021) & $0.14(0.05,0.37)$ & 17.26 \\
\hline li xiaoning (2019) & $0.15(0.04,0.59)$ & 6.94 \\
\hline Zhao xinping (2020) & $0.19(0.05,0.62)$ & 8.01 \\
\hline Zou yan (2017) & $0.16(0.05,0.53)$ & 9.19 \\
\hline Lu yuqing (2018) & $0.19(0.04,0.93)$ & 5.13 \\
\hline Li tingting (2018) & $0.23(0.08,0.68)$ & 9.29 \\
\hline Yu fang (2015) & $0.17(0.04,0.81)$ & 5.79 \\
\hline Tong yuehong (2017) & $0.07(0.01,0.60)$ & 5.93 \\
\hline liu miaomiao (2020) & $0.16(0.03,0.78)$ & 5.64 \\
\hline Clemens B Tempfer (2006) & $0.65(0.10,4.08)$ & 1.71 \\
\hline Arri Coomarasamy (2015) & $0.76(0.33,1.74)$ & 7.80 \\
\hline Ashok Kumar (2014) & $0.64(0.32,1.29)$ & 12.00 \\
\hline Muhammad Fawzy (2008) & $0.61(0.21,1.75)$ & 5.30 \\
\hline Overall $(I-$ squared $=41.3 \%, p=0.059$ ) & $0.30(0.22,0.40)$ & 100.00 \\
\hline
\end{tabular}

Figure 2 Meta-analysis of pregnancy success rate and adverse reaction rate after treatment in patients with recurrent spontaneous abortion. (A) Forest plot of pregnancy success rate; (B) forest plot of adverse reaction rate. 


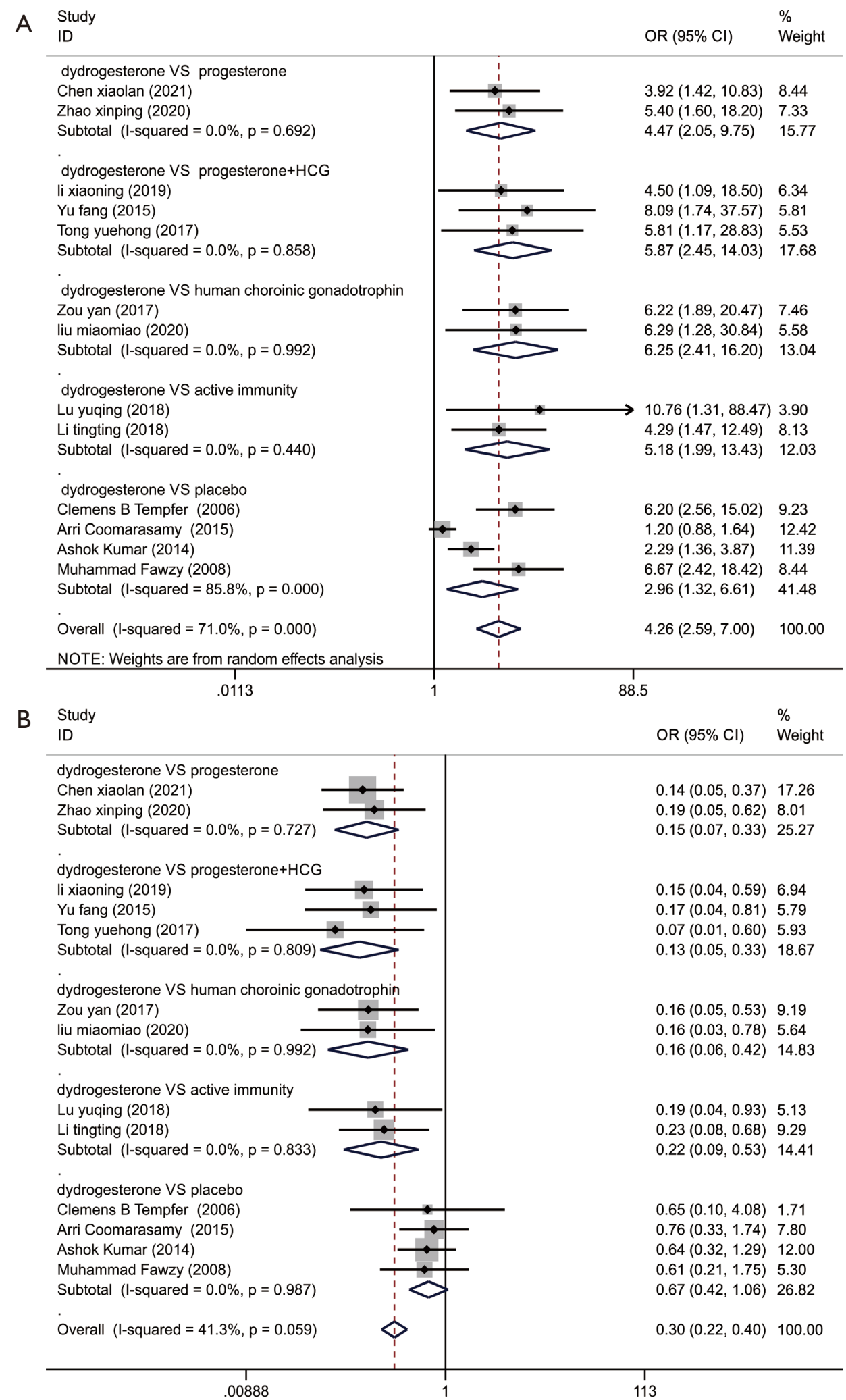

Figure 3 Subgroup analysis of pregnancy success rate and adverse reaction rate. (A) Subgroup analysis forest chart of pregnancy success rate; (B) subgroup analysis forest chart of adverse reaction rate. 
a placebo for the control group, and the heterogeneity between them was marked $\left(\mathrm{I}^{2}=85.8 \%, \mathrm{P}=0.000\right)$, and thus a random-effects model was selected. The results showed that the dydrogesterone group had higher a pregnancy success rate than the placebo group (OR $=2.96$; 95\% CI: $1.32-6.61$, $\mathrm{P}=0.008$ ).

In addition, we performed subgroup analysis of the adverse reactions of the different control group interventions (Figure 3B). Heterogeneity among the groups was not significant $\left(\mathrm{I}^{2}=0.0 \%, \mathrm{P}=0.727\right)$, and thus we chose the fixed-effect model for analysis. The results of 2 studies $(27,29)$ indicated that the incidence of adverse reactions when using dydrogesterone was lower than using progesterone alone (OR $=0.15$; $95 \%$ CI: 0.07 $0.33, \mathrm{P}=0.000)$. Three studies $(23,24,30)$ confirmed that the incidence of adverse reactions for dydrogesterone was lower than progesterone combined with hCG treatment ( $\mathrm{OR}=0.13 ; 95 \% \mathrm{CI}: 0.05-0.33, \mathrm{P}=0.000)$, and another 2 studies $(22,28)$ showed that the experimental group had a lower adverse reaction rate than hCG treatment alone (OR $=0.16$; 95\% CI: 0.06-0.42, $\mathrm{P}=0.000)$. Further, 2 studies $(25,26)$ showed that the dydrogesterone group had a lower adverse reaction rate than the active immunization group $(\mathrm{OR}=0.22$; 95\% CI: 0.09-0.53, $\mathrm{P}=0.000)$. The remaining 4 studies $(14,19-21)$ showed that the adverse reaction rate of the dydrogesterone group was lower than the placebo group $(\mathrm{OR}=0.67 ; 95 \%$ CI: $0.42-1.06, \mathrm{P}=0.089$ ).

\section{Sensitivity analysis of pregnancy success rate and adverse reaction rate}

Stata 16 software was used for sensitivity analysis to find heterogeneous sources to ensure the reliability of the results. By showing that the combined results of the included studies had little change and the sensitivity was low, we were able to confirm that the pregnancy success rate and the adverse reaction rate results were reliable (Figure $4 A, 4 B$ ).

\section{Publication bias analysis of pregnancy success rate and adverse reaction rate}

We then conducted publication bias analysis of the pregnancy success rate and adverse reaction rate of the 13 studies included in the meta-analysis. The asymmetric results (Figure $5 A, 5 B$ ) of the scatter distribution indicated an uneven sample distribution, suggesting that the studies may have had a certain degree of publication bias. This may have been due to a small sample size or low quality of research in some studies.

\section{Hormone levels after treatment}

Five studies $(23,24,26,27,30)$ contained outcome indicators showing changes in progesterone and hCG levels after receiving treatment for RSA. There was heterogeneity among these studies $\left(\mathrm{I}^{2}=87.1 \%, \mathrm{P}=0.000\right)$ and thus the results were combined and analyzed using a randomeffects model. The results revealed that the progesterone levels of RSA patients in the experimental group were significantly higher than those in the control group after treatment $(\mathrm{SMD}=1.93 ; 95 \% \mathrm{CI}: 1.38-2.48, \mathrm{P}=0.000$ ) (Figure 6A). Analysis of hCG levels after treatment showed dramatic heterogeneity between the studies $\left(\mathrm{I}^{2}=98.5 \%\right.$, $\mathrm{P}=0.000)$, revealing that the experimental group had a higher hCG level than the control group ( $\mathrm{SMD}=6.32$; 95\% CI: 3.58-9.07, $\mathrm{P}=0.000$ ) (Figure 6B), and therefore, a random-effects model was selected.

In order to ensure the reliability of the analysis, further sensitivity analysis was performed to find the source of heterogeneity. The sensitivity analysis showed that in the 5 studies involving treatment with dydrogesterone, the study by Li et al. (30) had a greater impact (Figure $7 A$ ) on the heterogeneity. Sensitivity analysis of hCG after treatment is shown in Figure 7B, which suggests that the study by $\mathrm{Lu}$ et al. (26) had a greater impact on heterogeneity.

\section{Immune factor levels after treatment}

In order to further evaluate the changes in the levels of immune factors after dydrogesterone treatment for RSA, we conducted a meta-analysis on the levels of IL-10, IL4 , and IFN- $\gamma$ after treatment. Among them, 5 studies $(20,25,28-30)$ detected the expression level of IL-10, and as the heterogeneity among the studies was significant $\left(\mathrm{I}^{2}=98.3 \%, \mathrm{P}=0.000\right)$, a random-effects model was selected. The results showed that the posttreatment level of IL-10 in the experimental group was higher than the control group ( $\mathrm{SMD}=2.01 ; 95 \%$ CI: 0.45-3.57, $\mathrm{P}=0.000$ ) (Figure $8 A$ ). In 5 studies $(20,22,23,28,29)$, random-effects model analysis $\left(\mathrm{I}^{2}=94.7 \%, \mathrm{P}=0.000\right)$ confirmed that after treatment, the experimental group had higher IL-4 levels than the control group ( $\mathrm{SMD}=1.12$; 95\% CI: 0.34-1.90, $\mathrm{P}=0.000$ ) (Figure $8 B$ ). There were 7 studies reporting the expression level of IFN- $\gamma$ after dydrogesterone treatment $(20,22,23,25,28-30)$, and the meta-analysis results $\left(\mathrm{I}^{2}=86.2 \%, \mathrm{P}=0.000\right)$ suggested that the experimental group had a notably lower IFN- $\gamma$ level than the control group after treatment (SMD $=-1.04 ; 95 \%$ CI: $-1.45-$ $-0.63, \mathrm{P}=0.000$ ) (Figure 8C).

To further verify these findings, we conducted another sensitivity analysis. The sensitivity analysis results of 


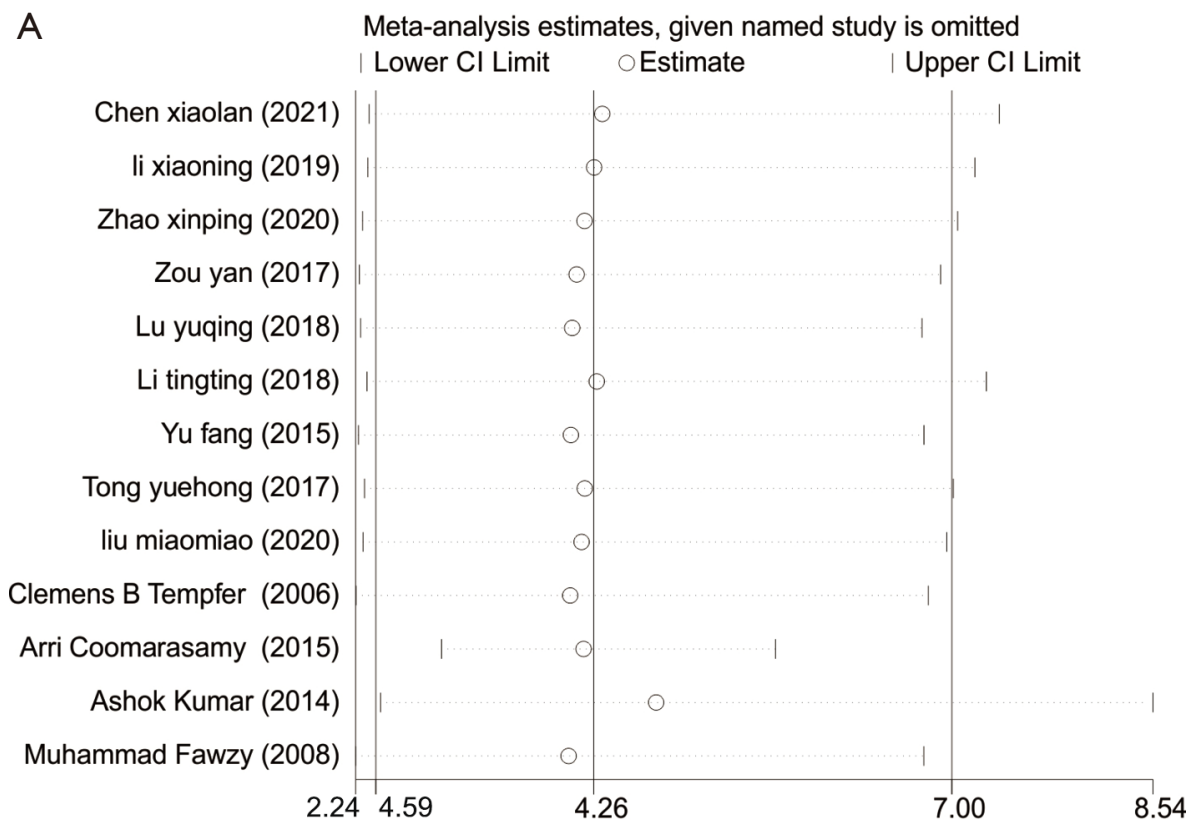

B

Meta-analysis estimates, given named study is omitted

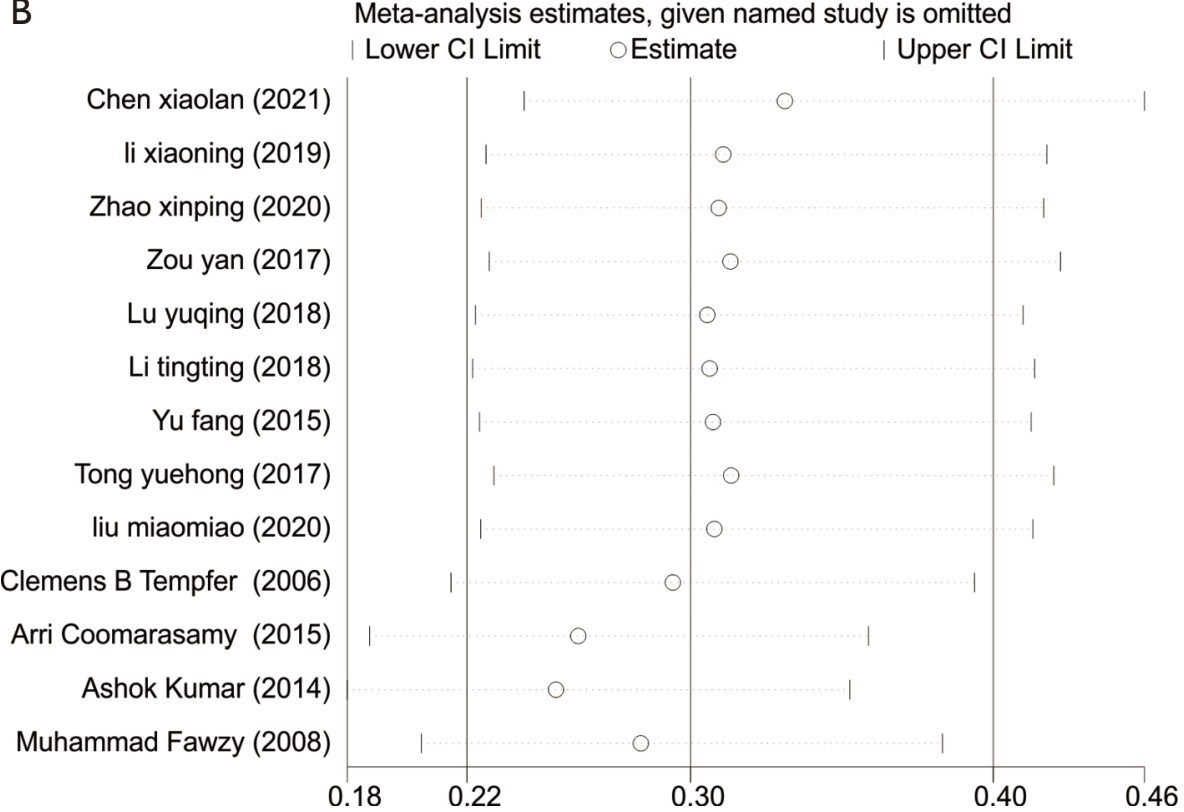

Figure 4 Sensitivity analysis. (A) Sensitivity analysis of pregnancy success rate; (B) sensitivity analysis of adverse reaction rate.

posttreatment IL-10 levels are shown in Figure 9A. The study by Kumar et al. (20) greatly influenced heterogeneity. Sensitivity analysis of IL-4 levels also suggested that the study by Kumar et al. (20) greatly impacted heterogeneity (Figure 9B). In addition, sensitivity analysis of posttreatment IFN- $\gamma$ levels is shown in Figure $9 C$, indicating that the study by Li et al. (30) had an impact on heterogeneity.

\section{Discussion}

Throughout a pregnancy, the mother's body must continuously sustain the normal growth of the embryo or fetus, and immune tolerance between the fetus and the mother is essential in determining the success of the pregnancy (31). The progesterone secreted by the corpus 

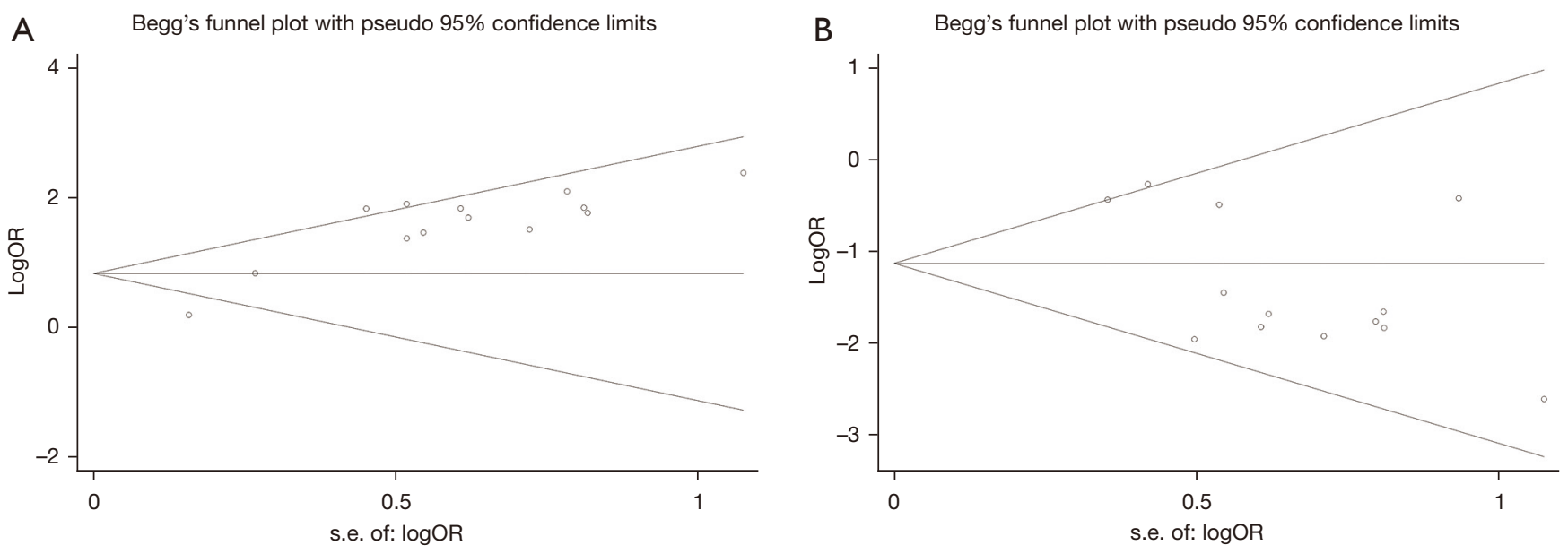

Figure 5 Publication bias analysis of pregnancy success rate and adverse reaction rate. (A) Funnel chart of pregnancy success rate; (B) funnel chart of adverse reaction rate.

luteum in the mother regulates the entire pregnancy process and also inhibits the immune response system at the maternal-fetal interface (32). Progesterone is secreted by the ovaries and placenta and acts on the endometrium and uterus to promote embryo implantation to protect pregnancy while also preventing and treating RSA through immune regulation $(13,33)$. The main role of the gonadotropin hCG is to maintain the secretion of progesterone and estrogen to form the decidua of the uterus, thereby promoting the growth of the placenta (34). As the reverse structure of progesterone, dydrogesterone has the same effects as progesterone, without adrenal cortex hormones or sex hormones. Further, dydrogesterone has high oral bioavailability and fewer adverse reactions and is thus widely used in the clinical treatment of RSA $(16,18)$. The side effects of didroxyprogesterone are less, with few reports of vaginal bleeding during treatment, and there are no reports of serious adverse reactions such as teratogenesis, which has high safety (30). Our meta-analysis found that after treating RSA patients with dydrogesterone, the pregnancy success rate was significantly higher than with progesterone, hCG, placebo, or active immunity, with a markedly lower adverse reaction rate as well. Further, after treatment with dydrogesterone, levels of progesterone and hCG in RSA patients were notably higher than those in the control group. These results indicated that after using dydrogesterone, RSA patients could become pregnant again.

Previous studies have shown that dydrogesterone can induce lymphocytes to produce progesterone, which upregulates Th2 cytokines in T helper cells (Th) and downregulates Th1 cytokines to induce blocking antibodies to protect against miscarriage $(35,36)$. IL-4 and IL-10 are Th2 type cytokines, and IFN- $\gamma$ is a typical Th1 type cytokine. The response of Th2 and Th1 cytokines to the trophoblast leads to the success and failure of pregnancy, respectively. Laskarin et al. (37) reported that the progesterone-induced blocking factor may participate in the immune response by regulating progesterone at the maternal-fetal interface to maintain pregnancy. Choi et al. (38) showed that the dosage of progesterone that can cause immunosuppression may be beneficial to the etiology of RSA and RSA patients who are Th1 dominant. Raghupathy et al. (39) extracted and separated monocytes from the blood of patients in vitro, then activated the immune response and cocultured with dydrogesterone, finding that the level of IFN- $\gamma$ decreased significantly, while the levels of IL-4 and IL-6 were increased. In the present study, after treatment with dydrogesterone, the levels of IL-4 and IL-6 in RSA patients increased significantly compared to the control group, while the level of IFN- $\gamma$ decreased dramatically. These results revealed that dydrogesterone effectively regulated and promoted the production of progesterone-induced blocking factors by lymphocytes to regulate the immune function of the maternal-fetal interface, thereby increasing the pregnancy success rate of RSA patients.

\section{Conclusions}

In summary, dydrogesterone had an obvious therapeutic 
A Study

ID

Chen xiaolan (2021)

li xiaoning (2019)

Lu yuqing (2018)

Yu fang (2015)

Tong yuehong (2017)

Overall (I-squared $=87.1 \%, p=0.000)$

NOTE: Weights are from random effects analysis
B Study

ID

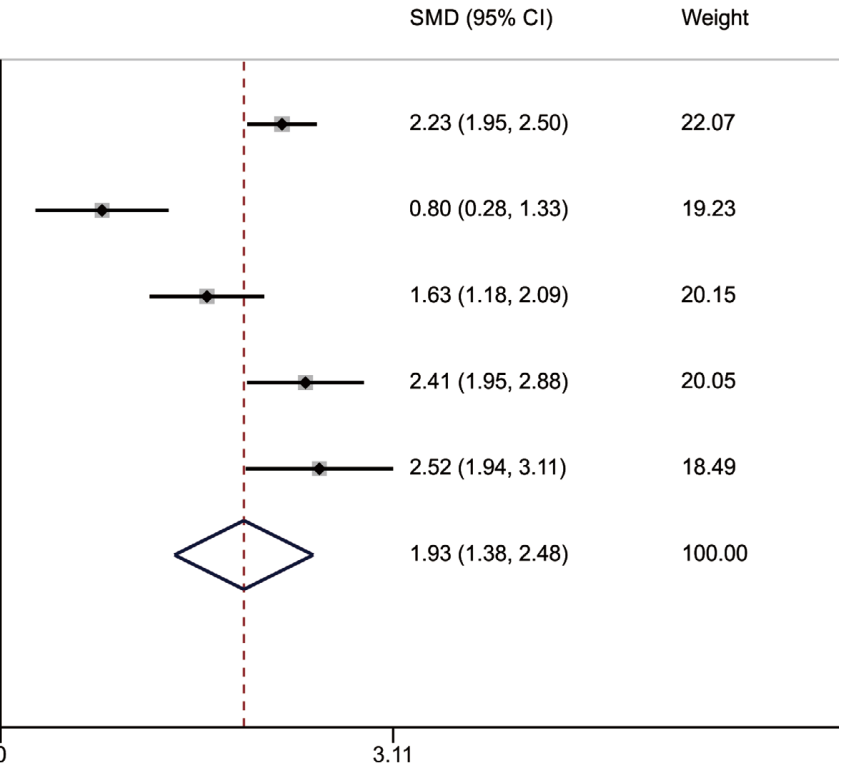

\begin{tabular}{|c|c|c|c|}
\hline Chen xiaolan (2021) & + & $6.38(5.85,6.92)$ & 20.46 \\
\hline li xiaoning (2019) & $\rightarrow$ & $4.17(3.26,5.09)$ & 20.16 \\
\hline Lu yuqing (2018) & & $13.40(11.49,15.32)$ & 18.73 \\
\hline Yu fang (2015) & & $6.50(5.62,7.39)$ & 20.18 \\
\hline Tong yuehong (2017) & $\star$ & $1.73(1.22,2.24)$ & 20.47 \\
\hline Overall $($ I-squared $=98.5 \%, p=0.000)$ & & $6.32(3.58,9.07)$ & 100.00 \\
\hline NOTE: Weights are from random effects analysis & & & \\
\hline
\end{tabular}

Figure 6 Forest plot of hormone levels after treatment of recurrent miscarriage. (A) Forest plot of progesterone levels after treatment; (B) forest plot of hCG levels after treatment. hCG, human chorionic gonadotropin.

effect on patients with unexplained RSA. It effectively improved levels of hCG and progesterone through immune regulation as well as the expression of IL-4, IL-10, and IFN- $\gamma$ cellular immune factors. Oral dydrogesterone is a safe and efficient treatment option for RSA patients and it can be applied clinically. To ensure that research conclusions are accurate and reliable, the standardization of clinical research should be improved, with more rigorously 

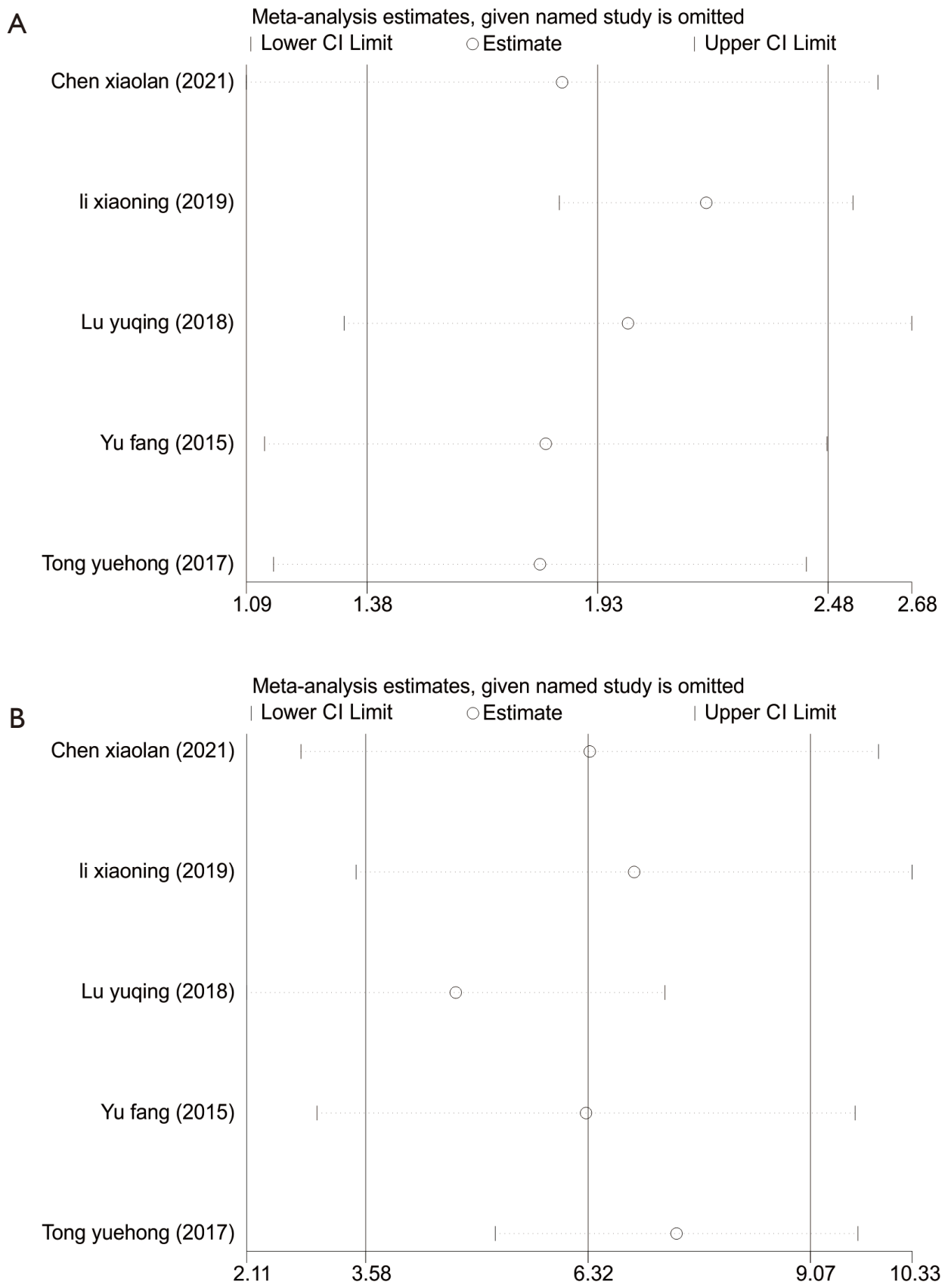

Figure 7 Sensitivity analysis of hormone levels after treatment of recurrent miscarriage. (A) Sensitivity analysis of progesterone levels after treatment; (B) sensitivity analysis of hCG levels after treatment. hCG, human chorionic gonadotropin. 


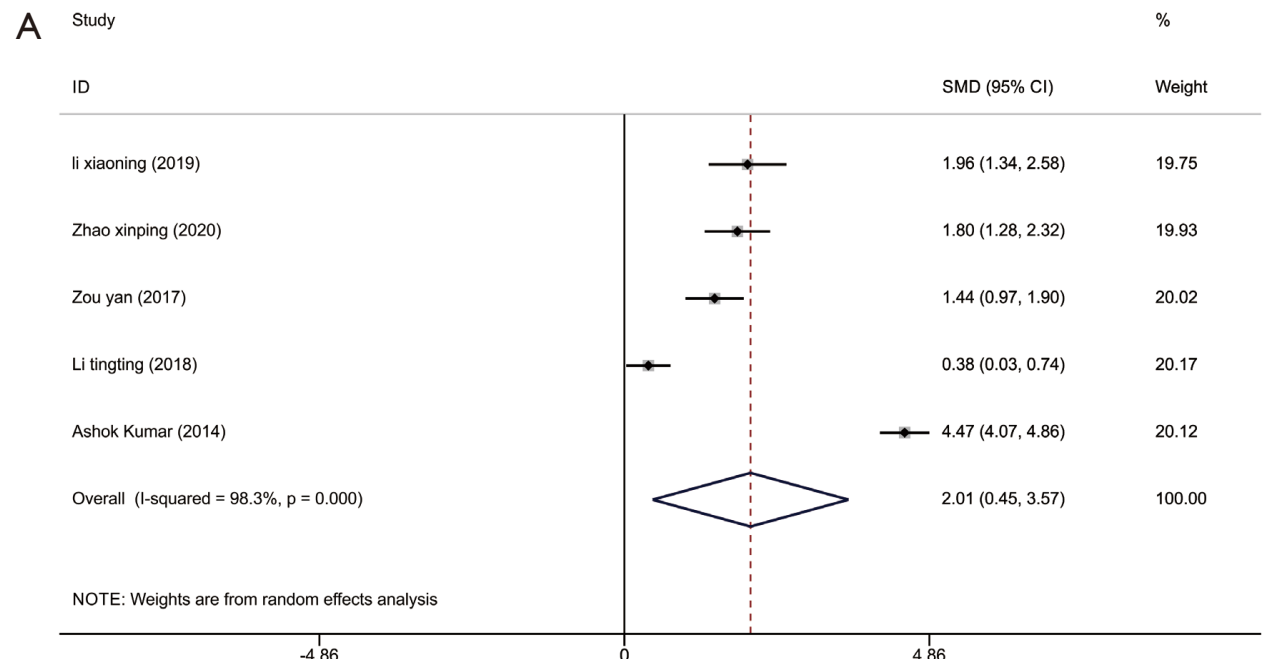

4.86

\section{$B$}

ID

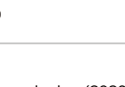

Zhao xinping (2020)

Zou yan (2017)

Tong yuehong (2017)

liu miaomiao (2020)

Ashok Kumar (2014)

Overall (1-squared $=94.7 \%, p=0.000)$

NOTE: Weights are from random effects analysis

NOTE: Weights are from random effects analysis

$\operatorname{SMD}(95 \% \mathrm{Cl})$

$\%$

C Study

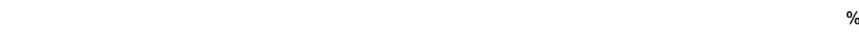

$\operatorname{SMD}(95 \% \mathrm{Cl}) \quad$ Weight

li xiaoning (2019)

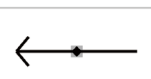

Zhao xinping (2020)

Zou yan (2017)

Li tingting (2018)

Tong yuehong (2017)

liu miaomiao (2020)

Ashok Kumar (2014)

Overall (I-squared $=86.2 \%, p=0.000)$

NOTE: Weights are from random effects analysis

$-3.81$

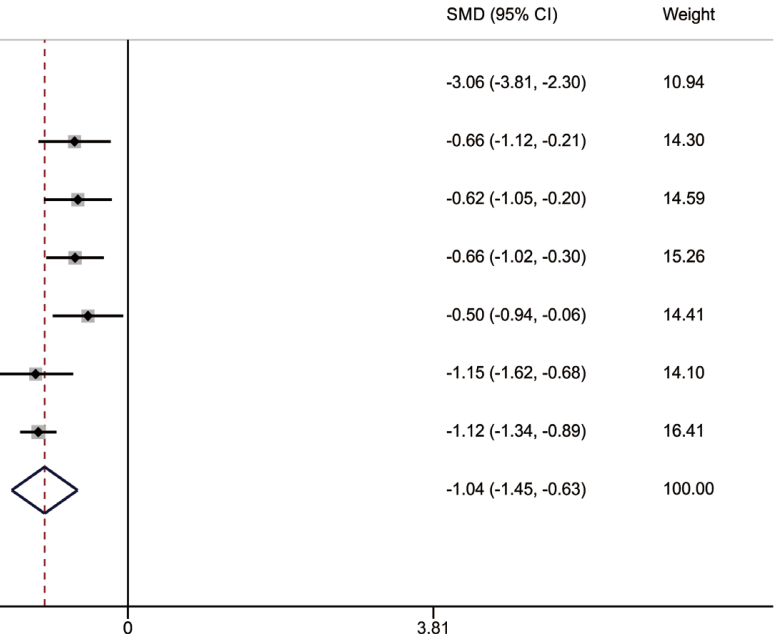

Figure 8 Forest plot of immune factor levels after treatment of recurrent miscarriage. (A) IL-10 level forest plot after treatment; (B) IL-4 level forest plot after treatment; (C) IFN- $\gamma$ level forest plot after treatment. IL-10, interleukin 10; IL-4, interleukin 4; IFN- $\gamma$, interferon-gamma. 
A

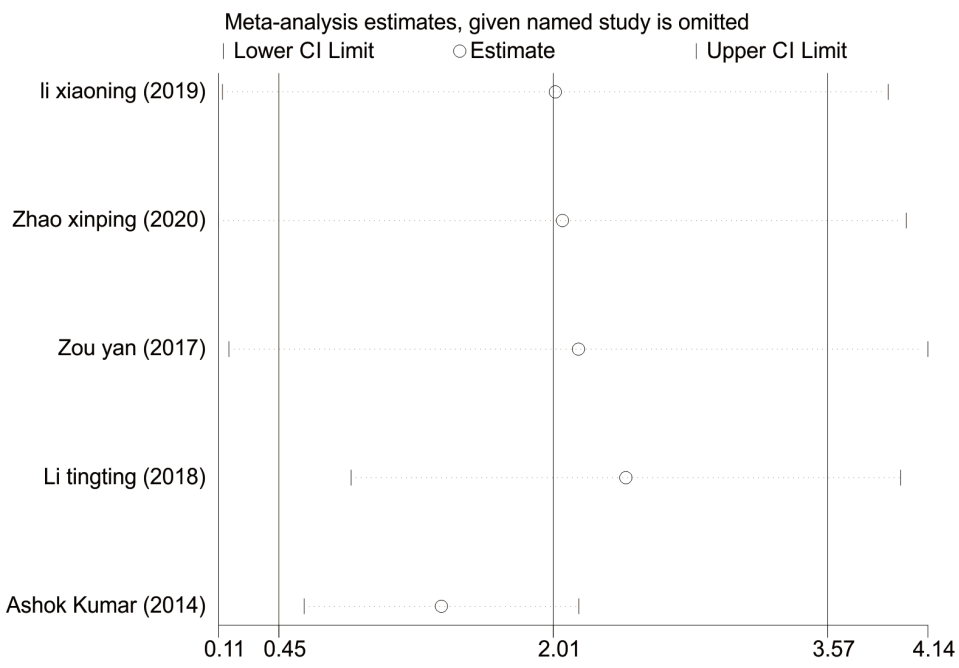

B

Meta-analysis estimates, given named study is omitted | Lower Cl Limit | Upper Cl Limit

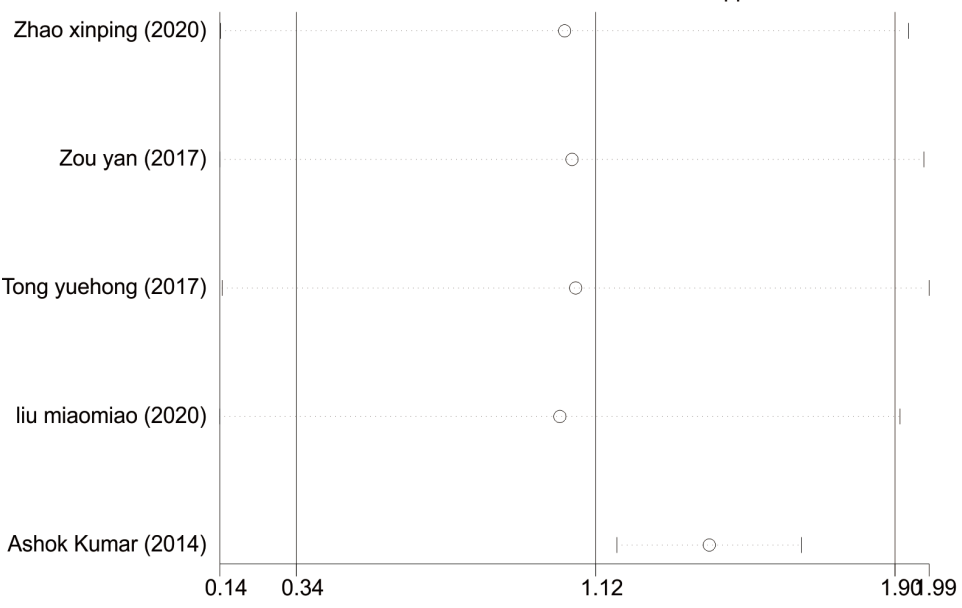

C

Meta-analysis estimates, given named study is omitted

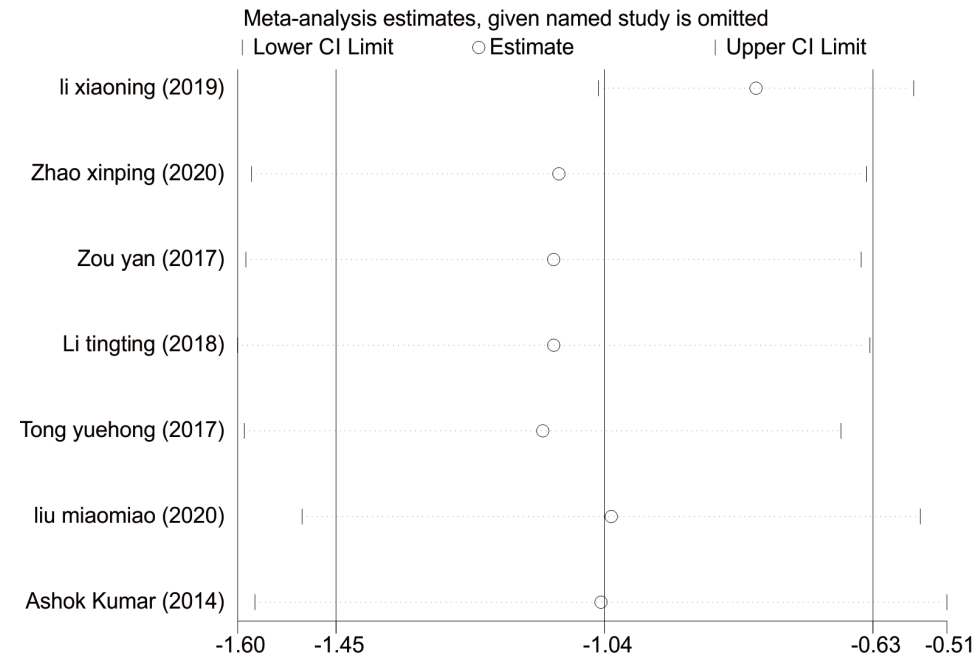

Figure 9 Sensitivity analysis of immune factor levels after treatment of recurrent miscarriage. (A) Sensitivity analysis of IL-10 level after treatment; (B) sensitivity analysis of IL-4 level after treatment; (C) sensitivity analysis of IFN- $\gamma$ level after treatment. IL-10, interleukin 10; IL-4, interleukin 4; IFN- $\gamma$, interferon-gamma. 
designed and high-quality studies carried out, while publication bias should be avoided as much as possible.

\section{Acknowledgments}

Funding: None.

\section{Footnote}

Reporting Checklist: The authors have completed the PRISMA reporting checklist. Available at https://dx.doi. org/10.21037/apm-21-2605

Conflicts of Interest: Both authors have completed the ICMJE uniform disclosure form (available at https://dx.doi. org/10.21037/apm-21-2605). Both authors have no conflicts of interest to declare.

Ethical Statement: The authors are accountable for all aspects of the work in ensuring that questions related to the accuracy or integrity of any part of the work are appropriately investigated and resolved.

Open Access Statement: This is an Open Access article distributed in accordance with the Creative Commons Attribution-NonCommercial-NoDerivs 4.0 International License (CC BY-NC-ND 4.0), which permits the noncommercial replication and distribution of the article with the strict proviso that no changes or edits are made and the original work is properly cited (including links to both the formal publication through the relevant DOI and the license). See: https://creativecommons.org/licenses/by-nc-nd/4.0/.

\section{References}

1. Compilation group of Chinese expert consensus on the diagnosis and treatment of spontaneous abortion. Chinese Expert Consensus on the Diagnosis and Treatment of Spontaneous Abortion (2020 Edition). Chinese Journal of Practical Gynecology and Obstetrics 2020;36:1082-90.

2. Obstetrics Subgroup, Chinese Society of Obstetrics and Gynecology, Chinese Medical Association. Chinese expert consensus on the diagnosis and tretment of recurrent spontaneous abortion. Zhonghua Fu Chan Ke Za Zhi 2016;51:3-9.

3. Coomarasamy A, Dhillon-Smith RK, Papadopoulou A, et al. Recurrent miscarriage: evidence to accelerate action. Lancet 2021;397:1675-82.
4. Green DM, O'Donoghue K. A review of reproductive outcomes of women with two consecutive miscarriages and no living child. J Obstet Gynaecol 2019;39:816-21.

5. Nybo Andersen AM, Wohlfahrt J, Christens P, et al. Maternal age and fetal loss: population based register linkage study. BMJ 2000;320:1708-12.

6. Regan L, Braude PR, Trembath PL. Influence of past reproductive performance on risk of spontaneous abortion. BMJ 1989;299:541-5.

7. Practice Committee of the American Society for Reproductive Medicine. Evaluation and treatment of recurrent pregnancy loss: a committee opinion. Fertil Steril 2012;98:1103-11.

8. Wong LF, Porter TF, Scott JR. Immunotherapy for recurrent miscarriage. Cochrane Database Syst Rev 2014;(10):CD000112.

9. Saccone G, Schoen C, Franasiak JM, et al. Supplementation with progestogens in the first trimester of pregnancy to prevent miscarriage in women with unexplained recurrent miscarriage: a systematic review and meta-analysis of randomized, controlled trials. Fertil Steril 2017;107:430-438.e3.

10. Mller KA, Fuchs F. Double-blind controlled trial of 6methyl, 17-acetoxyprogesterone in threatened abortion. BJOG 1965;72:1042-44.

11. EPPPIC Group. Evaluating Progestogens for Preventing Preterm birth International Collaborative (EPPPIC): meta-analysis of individual participant data from randomised controlled trials. Lancet 2021;397:1183-94.

12. Stanczyk FZ, Hapgood JP, Winer S, et al. Progestogens used in postmenopausal hormone therapy: differences in their pharmacological properties, intracellular actions, and clinical effects. Endocr Rev 2013;34:171-208.

13. Walch K, Hefler L, Nagele F. Oral dydrogesterone treatment during the first trimester of pregnancy: the prevention of miscarriage study (PROMIS). A double-blind, prospectively randomized, placebo-controlled, parallel group trial. J Matern Fetal Neonatal Med 2005;18:265-9.

14. Tempfer CB, Kurz C, Bentz EK, et al. A combination treatment of prednisone, aspirin, folate, and progesterone in women with idiopathic recurrent miscarriage: a matched-pair study. Fertil Steril 2006;86:145-8.

15. Schindler AE. Progestational effects of dydrogesterone in vitro, in vivo and on the human endometrium. Maturitas 2009;65 Suppl 1:S3-11.

16. Mirza FG, Patki A, Pexman-Fieth C. Dydrogesterone use in early pregnancy. Gynecol Endocrinol 2016;32:97-106.

17. Raghupathy R, Kalinka J. Cytokine imbalance in 
pregnancy complications and its modulation. Front Biosci 2008;13:985-94.

18. Gruber CJ, Huber JC. The role of dydrogesterone in recurrent (habitual) abortion. J Steroid Biochem Mol Biol 2005;97:426-30.

19. Fawzy M, Shokeir T, El-Tatongy M, et al. Treatment options and pregnancy outcome in women with idiopathic recurrent miscarriage: a randomized placebo-controlled study. Arch Gynecol Obstet 2008;278:33-8.

20. Kumar A, Begum N, Prasad S, et al. Oral dydrogesterone treatment during early pregnancy to prevent recurrent pregnancy loss and its role in modulation of cytokine production: a double-blind, randomized, parallel, placebocontrolled trial. Fertil Steril 2014;102:1357-1363.e3.

21. Coomarasamy A, Williams H, Truchanowicz E, et al. A Randomized Trial of Progesterone in Women with Recurrent Miscarriages. N Engl J Med 2015;373:2141-8.

22. Liu MM, Zhang L. Comparison on the Efficacy of Dermoprogesterone Tablets and Chorinonic Gonadotrophin for Injection in the Treatment of Unexplained Recurrent Spontaneous Abortion. Clinical Medical and Engineering 2020;27:1021-2.

23. Tong YH, Shao MJ, Hu M, et al. Regulatory effect of dydrogesterone tablets combined with HCG on the level of hormone and cytokines of women with recurrent spontaneous abortion. Chinese Journal of Family Planning 2017;25:21-4.

24. Yu F, Jia HJ, Liao YF. A Comparison of the Clinical efifcacy of Dydrogesterone and Progesterone in the Treatment of Unexplained Recurrent Spontaneous Abortion. Journal of Rare and Uncommon Diseases 2015;22:33-5.

25. Li TT, Luo ZH, Yao JL, et al. Influence of Active Immunization Combined with Progesterone Treatment on Antinuclear Antibodies in Patients with Unexplained Recurrent Spontaneous Abortion. Clinical Medical and Engineering 2018;25:627-8.

26. Lu YQ. To observe the efficacy and safety of dydrogesterone assisted active immunotherapy in the treatment of recurrent miscarriage. China Practical Medical 2018;13:138-9.

27. Chen XL. Analysis of the Effect of Dydrogesterone on Patients with Recurrent Miscarriage. Systems Medicine 2021;6:137-40.

28. Zou Y, Zhang M. Effects of Progesterone Tablets in the Patients with Unexplained Recurrent Spontaneous Abortion and Influence on Cellular Immunity Factor. China Pharmacist 2017;20:1243-5.

29. Zhao XP, Wang TT, Zhang AQ, et al. Observation on the therapeutic effect of didroxyprogesterone tablets on patients with unexplained recurrent abortion. Chinese Community Doctors 2020;36:63-4.

30. Li XN, Zhang PY, Hui XL. Comparison of the effect of dydrogesterone and progesterone in unexplained recurrent miscarriage. Guizhou Medical Journal 2020;44:1739-40.

31. Ali S, Majid S, Ali MN, et al. Cytokine imbalance at materno-embryonic interface as a potential immune mechanism for recurrent pregnancy loss. Int Immunopharmacol 2021;90:107118.

32. Shah NM, Lai PF, Imami N, et al. Progesterone-Related Immune Modulation of Pregnancy and Labor. Front Endocrinol (Lausanne) 2019;10:198.

33. Raghupathy R, Al Mutawa E, Makhseed M, et al. Modulation of cytokine production by dydrogesterone in lymphocytes from women with recurrent miscarriage. BJOG 2005;112:1096-101.

34. de Ziegler D, Pirtea P, Andersen CY, et al. Role of gonadotropin-releasing hormone agonists, human chorionic gonadotropin (hCG), progesterone, and estrogen in luteal phase support after hCG triggering, and when in pregnancy hormonal support can be stopped. Fertil Steril 2018;109:749-55.

35. Kelemen K, Bognar I, Paal M, et al. A progesteroneinduced protein increases the synthesis of asymmetric antibodies. Cell Immunol 1996;167:129-34.

36. Druckmann R, Druckmann MA. Progesterone and the immunology of pregnancy. J Steroid Biochem Mol Biol 2005;97:389-96.

37. Laskarin G, Tokmadzić VS, Strbo N, et al. Progesterone induced blocking factor (PIBF) mediates progesterone induced suppression of decidual lymphocyte cytotoxicity. Am J Reprod Immunol 2002;48:201-9.

38. Choi BC, Polgar K, Xiao L, et al. Progesterone inhibits invitro embryotoxic Th1 cytokine production to trophoblast in women with recurrent pregnancy loss. Hum Reprod 2000;15 Suppl 1:46-59.

39. Raghupathy R, Al Mutawa E, Makhseed M, et al. Redirection of cytokine production by lymphocytes from women with pre-term delivery by dydrogesterone. Am J Reprod Immunol 2007;58:31-8.

(English Language Editor: A. Muijlwijk)

Cite this article as: Guo H, Lu Q. Efficacy of dydrogesterone on treating recurrent miscarriage and its influence on immune factors: a systematic review and meta-analysis. Ann Palliat Med 2021;10(10):10971-10985. doi: 10.21037/apm-21-2605 